\title{
Demystifying dyssynchrony for diagnosis and prognosis: Tips for measuring heterogeneous phase distribution
}

\author{
Kenichi Nakajima, MD, PhD, ${ }^{a}$ Koichi Okuda, $\mathrm{PhD},{ }^{\mathrm{b}}$ and Koji Maruyama, $\mathrm{PhD}^{\mathrm{c}, \mathrm{d}}$ \\ a Department of Nuclear Medicine/Functional Imaging and Artificial Intelligence, Kanazawa \\ University, Kanazawa, Japan \\ b Department of Physics, Kanazawa Medical University, Kahoku, Japan \\ c Wolfram Research Inc., Tokyo, Japan \\ d Department of Chemistry and Materials Science, Osaka City University, Osaka, Japan
}

Received Aug 4, 2019; accepted Aug 5, 2019

doi: 10.1007/s12350-019-01855-0

\section{See related article, pp. 1055-1063}

Scintigraphic patterns can be visually assessed with reasonable accuracy. For example, radionuclide distribution on a scintigraphic image can easily be visually defined as heterogeneous. However, the quantitation of heterogeneity on nuclear medicine images has remained an important and complex issue. Region-of-interest analysis that provides mean, maximum, minimum, and standard deviation (SD) is the most popular means of evaluating regional data, but these variables do not reflect all image features, and minor abnormalities in distribution cannot be precisely quantified using these conventional variables. Consider a situation where minor changes in heterogeneous distribution can be visually identified on myocardial perfusion images of patients with cardiomyopathy. For example, the average of individual segments in a 17-segment model of polar maps cannot so clearly identify an obvious visible abnormality.

Reprint requests: Kenichi Nakajima, MD, PhD, Department of Nuclear Medicine/Functional Imaging and Artificial Intelligence, Kanazawa University, 13-1 Takara-machi, Kanazawa920-8640, Japan; nakajima@med.kanazawa-u.ac.jp

J Nucl Cardiol 2021;28:1064-7.

$1071-3581 / \$ 34.00$

Copyright (C) 2019 American Society of Nuclear Cardiology.

\section{VARIABLES FROM HISTOGRAM ANALYSIS}

Discrete Fourier transform is applied to temporal data pixel by pixel in phase analysis of gated myocardial perfusion to determine activity during cardiac cycles, and phase delay derived from a fundamental frequency is mapped on the polar coordinate. A histogram of phase values will show a single clear peak that corresponds to the timing of cardiac contraction (Figure 1). The mean and SD might reflect the characteristic histogram shape of a simultaneous contraction. On the other hand, when the histogram shows a more complicated structure, such as that with multiple peaks, the SD cannot reveal the characteristics of the distribution. The range between the maximum and minimum might be useful to assess such incoherent distribution. Nevertheless, the total range is likely to be affected by noise or outliers. Thus, the $95 \%$ width of the histogram might be more convenient to enhance the reproducibility and stability of the measure. However, since the $95 \%$ bandwidth is still determined only by data points ranging from $2.5 \%$ to $97.5 \%$ on the histogram, it does not reflect the characteristics of entire histogram structure. Similarly to the first and second order moments (mean and SD), other variables such as third and the fourth order moments can be calculated. ${ }^{1}$ The third order moment, skewness, is defined as $\left.\alpha_{3}=\Sigma\left[(X-\mu)^{3}\right]\right) / \sigma^{3}(\mu$, mean; $\sigma$, SD) and reflects the measure of symmetry. When $\alpha_{3}<0$ and $>0$, the left and right tails of the histogram are respectively longer. The fourth order moment, kurtosis, is defined as $\left.\alpha_{4}=\Sigma\left[(X-\mu)^{4}\right]\right) / \sigma^{4}-3$ and measures the sharpness of the peak. When $\alpha_{4} *<0$ and $>0$, the peak will be respectively more round and more acute than normal distribution (*standard normal distribution has a kurtosis of zero). Although these higher-order moments can easily be calculated, clinical applications have not yet been clarified. 


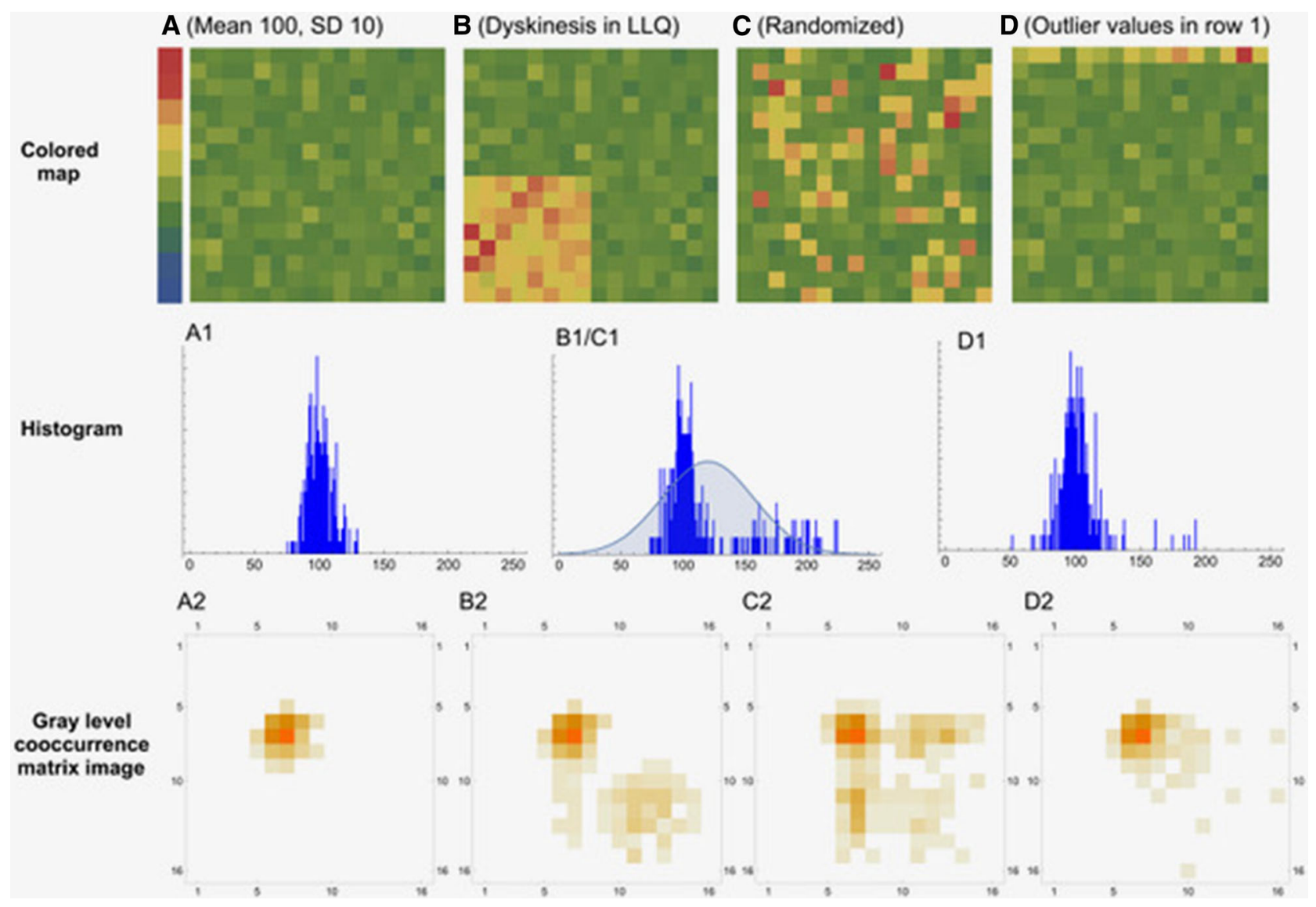

Figure 1. Sample models of phase images, histograms and texture analysis. Map $(16 \times 16$ pixels $)$ with mean $=100$ and standard deviation $(\mathrm{SD})=10(\mathbf{A})$. Delayed phase values are localized in left lower quadrant (LLQ) (B); and same values are randomized in location (C). In panel $\mathbf{D}$, outlier values are added to row 1. Histogram A1 is created from image A, histogram in panels B1/C1 is created from maps of both $\mathbf{B}$ and $\mathbf{C}$, and normal distribution with same mean and SD overlaps. Histogram in panel D1 is created from map D. Bottom row, co-occurrence matrix maps for each image.

The order and disorder of distribution can also be quantified by entropy, namely $\left.-\Sigma f_{i} \times \log \left(f_{i}\right)\right] / \log (\mathrm{n})$, which is normalized by $\log (\mathrm{n})$ to bring its range to $[0,1]$. In this definition, $f(i)$ is the frequency of the histogram of each bin, namely the probability of being in the $i$ th bin. Entropy might be able to effectively characterize the heterogeneity or homogeneity of distributions in maps. ${ }^{2,3}$ This editorial examines the most common phase patterns and some fundamental factors influencing bandwidth, SD, and entropy.

\section{APPLICATION TO PATIENTS WITHOUT CORONARY ARTERY STENOSIS}

In this issue of the Journal of Nuclear Cardiology ${ }^{\circledR}$, Peix and colleagues described the use of gated myocardial perfusion SPECT for phase analyses of patients without significant coronary artery stenosis. ${ }^{4}$ They found that female patients without stenosis but with summed stress scores $>4$ (suggesting ischemia) had a higher prevalence of left ventricular dilation and reduced ejection fraction compared with non-ischemic patients. The phase SD and bandwidth were also larger in this group. The tendency was similar among males, although the statistical significance was lower than in females. This finding means that heterogeneous contraction time was induced in ischemic patients, even in those without coronary artery stenosis. Although minor changes in conventional parameters might not be obvious, dyssynchrony variables could reflect underlying pathophysiological changes. 


\section{REGIONAL VS DIFFUSE ABNORMALITY}

The study by Peix and colleagues did not determine the nature of the changes in the distribution of phase polar maps ${ }^{4}$; two explanations could account for this finding. One is when apparent regional ischemia subsequently causes stress-induced defects and reduced motion, and this results in a phase delay. Both regionally decreased counts and a reduced amplitude of wall motion will increase relative noise, and the variability of phase values will be augmented. ${ }^{5}$ This type of phase delay is more prevalent when some ischemic segments are severely hypokinetic, akinetic, and dyskinetic. Based on induced ischemia, patients with heart failure of ischemic and non-ischemic etiology could be differentiated by phase analysis. ${ }^{6}$ The prevalence of dyssynchrony associated with myocardial perfusion abnormalities is high regardless of systolic dysfunction. ${ }^{7}$ The other explanation is that even in the absence of an obvious localized abnormality, contraction on the entire myocardium might have become heterogeneous, and lead to widespread phase distribution. This could be associated with disturbed microcirculation in periphery of coronary arteries or the myocardium. Scattered fibrosis in the myocardium as found in primary and secondary cardiomyopathy, including chronic kidney disease and renal failure, might cause such widespread abnormalities. ${ }^{8,9}$ In these situations, activity might have become heterogeneously reduced and ventricles might have become dilated, which would increase relative noise in phase. Local and diffuse abnormalities can concomitantly affect heterogeneous phase distribution in clinical situations. Left ventricular volume, ejection fraction, and total counts during data acquisition affect phase variability even in healthy persons. ${ }^{5,10}$ A physiological difference between male and female hearts could be considered. Moreover, because phase variation is relatively smaller in females, probably due to higher counts in the unit myocardium, and a smaller heart than in males, then careful analysis will be required if study groups contain different numbers of males and females.

\section{SIMPLE MODELS TO UNDERSTAND PHASE VARIABLES}

To understand differences in distribution patterns, simple models of phase map values can be constructed as shown in Figure 1. We assigned random integer values between 0 and 255 to each pixel in a $16 \times 16$ pixel area. When these values are generated according to the normal distribution with the mean and SD being 100 and 10 , respectively, the $95 \%$ bandwidth, skewness, and kurtosis are calculated as in Table 1. If the histogram bin size is changed from 1 to 5 and 10 , all values are rounded to multiples of 5 and 10, respectively, and the statistical measures will accordingly change. Entropy is influenced by bin size, because values are classified into the same bins, that is, more homogeneity. Appropriate bin size is therefore important to obtain stable results. Skewness and kurtosis are changed according to changes in histogram shape.

Data representing dyskinesis that have been artificially added into the left lower quadrant of Figure 1B have increased the values for mean, SD, bandwidth $95 \%$, and entropy. Naturally, even if the values are randomized (Figure 1C), these values will be identical and the histogram will be the same (Figures 1B1/C1). In Figures $1 \mathrm{~B} 1 / \mathrm{C} 1$, the normal distribution with the same mean and SD of the overall data is superimposed, which vividly shows the limitation of using the simple phase SD.

Another caution needed for clinical application is outlier phase values that sometimes appear in peripheral parts of the map (Figure 1D). This is caused by a partial volume effect along the border of the valve plane, and the reduced count causes a larger variation in the phase. If only SD and bandwidth are considered, a larger SD and bandwidth can be calculated, but entropy is less

Table 1. Phase histogram parameters calculated from images in Figure 1

\begin{tabular}{llcccccc}
\hline Image & $\begin{array}{c}\text { Histogram bin or } \\
\text { image }\end{array}$ & Mean & $\begin{array}{c}\text { Standard } \\
\text { deviation }\end{array}$ & $\begin{array}{c}\text { Bandwidth } \\
\mathbf{9 5 \%}\end{array}$ & Entropy & Skewness & Kurtosis \\
\hline A & Histogram bin size $=1$ & 100 & 10 & 32 & 0.64 & 0.42 & 3.17 \\
& 5 & 98 & 10 & 30 & 0.52 & 0.37 & 3.00 \\
& 10 & 96 & 10 & 30 & 0.44 & 0.25 & 2.90 \\
B & Dyskinesis in LLQ & 120 & 37 & 108 & 0.74 & 1.28 & 3.34 \\
C & Randomized & 120 & 37 & 108 & 0.74 & 1.28 & 3.34 \\
D & Outlier values in row 1 & 102 & 17 & 43 & 0.67 & 2.35 & 12.8 \\
\hline
\end{tabular}

$L L Q$, left lower quadrant 
affected by such peripheral noise (Table 1). However, these outlier values should be excluded during processing to generate stable results. Counts could also be very low, for example, in an area of infarct with minimal uptake, but these areas could be interpreted as true findings.

\section{DYSSYNCHRONY ANALYSIS FOR FURTHER STUDIES}

Dyssynchrony indices can visualize variations in the timing of contraction that cannot be clearly discerned visually. Hence, phase-based dyssynchrony analysis should play further roles in diagnosis and prognosis. Although optimal cut-off values for diagnostic performance might differ among software packages, the diagnostic ability of phase bandwidth, SD, and entropy is acceptable for any software. ${ }^{11}$ As phase analysis can be readily added using standard tomographic myocardial perfusion images, dyssynchrony analysis might serve as routine data processing to evaluate regional and global contraction disturbances. Sometimes, even when regional abnormality is not clearly visualized, dyssynchrony parameters might provide new insights for diagnosis and prognosis. The characteristics of phase images and limitations need to be understood because these variables are common scalar values calculated from histogram distribution.

In order to investigate regional phase distribution patterns further, additional methods such as texture analysis may be applied. Figures 1A2-D2 shows simple models of a gray-level co-occurrence matrix with different patterns for images A, B, C, and D. In the matrix plot, the elements $m_{i j}$ represent the probability of all occurrences of a pixel with intensity $i$ to the left or bottom of a pixel with intensity $j$, assuming all pixels lie in one of $n$ successive bins. However, the clinical utility of texture analysis is yet to be determined.

The applicability of phase analysis to ischemic and non-ischemic cardiac diseases and the possibility of additional variables are under investigation until definite phase analysis indications are determined for diagnosis and prognosis. When factors influencing dyssynchrony variables are clarified, the results would be less mystifying, and they should become indispensable tools for nuclear cardiology.

\section{Acknowledgments}

All the simulation data and processing were created using Mathematica version 12.0 (Wolfram Research, Inc.). The authors thank Norma Foster for editorial assistance.

\section{Disclosure}

None.

\section{References}

1. Chen J, Garcia EV, Folks RD, Cooke CD, Faber TL, Tauxe EL, Iskandrian AE. Onset of left ventricular mechanical contraction as determined by phase analysis of ECG-gated myocardial perfusion SPECT imaging: Development of a diagnostic tool for assessment of cardiac mechanical dyssynchrony. J Nucl Cardiol 2005;12:68795.

2. O'Connell JW, Schreck C, Moles M, Badwar N, DeMarco T, Olgin J, Lee B, Tseng Z, Kumar U, Botvinick EH. A unique method by which to quantitate synchrony with equilibrium radionuclide angiography. J Nucl Cardiol 2005;12:441-50.

3. Van Kriekinge SD, Nishina H, Ohba M, Berman DS, Germano G. Automatic global and regional phase analysis from gated myocardial perfusion SPECT imaging: Application to the characterization of ventricular contraction in patients with left bundle branch block. J Nucl Med 2008;49:1790-7.

4. Peix A, Padron K, Cabrera LO, Pardo L, Sanchez J. Left ventricular mechanical dyssynchrony in patients with chest pain and normal epicardial coronary arteries. J Nucl Cardiol 2019. https://d oi.org/10.1007/s12350-019-01804-x Epub ahead of print.

5. Nakajima K, Okuda K, Matsuo S, Slomka P. Making the invisible visible: Phase dyssynchrony has potential as a new prognostic marker. J Nucl Cardiol 2019;26:298-302.

6. Igarashi Y, Chikamori T, Hida S, Tanaka H, Shiba C, Usui Y, Hatano T, Yamashina A. Usefulness of phase analysis to differentiate ischemic and non-ischemic etiologies of left ventricular systolic dysfunction in patients with heart failure. Circ J 2014;78:141-50.

7. Gimelli A, Liga R, Giorgetti A, Favilli B, Pasanisi EM, Marzullo P. Determinants of left ventricular mechanical dyssynchrony in patients submitted to myocardial perfusion imaging: A cardiac CZT study. J Nucl Cardiol 2016;23:728-36.

8. Aggarwal H, AlJaroudi WA, Mehta S, Mannon R, Heo J, Iskandrian AE, Hage FG. The prognostic value of left ventricular mechanical dyssynchrony using gated myocardial perfusion imaging in patients with end-stage renal disease. J Nucl Cardiol 2014;21:739-46.

9. Mori H, Isobe S, Suzuki S, Unno K, Morimoto R, Kano N, Okumura T, Yasuda Y, Kato K, Murohara T. Prognostic value of left ventricular dyssynchrony evaluated by gated-SPECT in CKD patients with normal perfusion defect scores. J Nucl Cardiol 2019;26:288-97.

10. Nakajima K, Okuda K, Matsuo S, Kiso K, Kinuya S, Garcia EV. Comparison of phase dyssynchrony analysis using gated myocardial perfusion imaging with four software programs: Based on the Japanese Society of Nuclear Medicine Working Group normal database. J Nucl Cardiol 2017;24:611-21.

11. Okuda K, Nakajima K, Matsuo S, Kashiwaya S, Yoneyama H, Shibutani T, Onoguchi M, Hashimoto M, Kinuya S. Comparison of diagnostic performance of four software packages for phase dyssynchrony analysis in gated myocardial perfusion SPECT. EJNMMI Res 2017;7:27.

Publisher's Note Springer Nature remains neutral with regard to jurisdictional claims in published maps and institutional affiliations. 